\title{
Dental Post
}

National Cancer Institute

\section{Source}

National Cancer Institute. Dental Post. NCI Thesaurus. Code C66863.

A rod that a dentist has positioned and then cemented in the canal space in a tooth's

root. 